\title{
Anticancer drug targets: growth factors and growth factor signaling
}

\author{
Jackson B. Gibbs \\ WP16-101 Cancer Research, Department of Cancer Research, Merck Research Laboratories, \\ Sumneytown Pike, West Point, Pennsylvania 19486, USA. \\ Phone: (215) 652-5278; Fax: (215) 652-7320; E-mail: jay_gibbs@merck.com.
}

Signaling mechanisms that drive cell proliferation are closely associated with tumor malignancy. Components of these pathways, encoded by some of the very first oncogenes identified, include the PDGF-like ligand Sis, the tyrosine kinases Src and HER-2/c-Neu (HER-2), and the GTP-binding switch Ras. The study of communication by these oncoproteins has identified a complex array of intracellular circuits. In some cancers, mutations in key components lead to constitutive activation of these pathways; this activation is associated with the proliferative properties of the tumor cells. In this Perspective, I provide a broad overview of a growth factor signal transduction system, with a focus on those points that have been translated to drugs or clinical candidates. Due to editorial restrictions limiting the number of reference citations, much of the clinical data gleaned from abstracts is not listed in the references. Instead, the reader is directed to the 1999 Proceedings of the American Society of Clinical Oncology and the 1999 Proceedings of the AACR-NCI-EORTC International Conference.

Signaling pathways are initiated with the binding of a ligand, such as PDGF, EGF, EGF-like ligands (e.g., TGF- $\alpha$ and amphiregulin), or IGF, to its cognate transmembrane receptor (1). Ligand binding induces the dimerization of receptor subunits, promoting autophosphorylation of the receptor and recruiting a variety of intracellular docking proteins (such as Grb2, Shc, and Nck) to the plasma membrane. These docking proteins create a molecular scaffold from which subsequent signals emanate. For example, the guanine nucleotide exchange factor Sos binds to Grb2, which in turn interacts with the Ras protein. Ras serves as a molecular switch in the plasma membrane that alternates between an inactive GDP-bound state and an active GTP-bound state. Normally, Ras is bound to GDP because of the abundance of GTPase-activating protein and neurofibromin, which both suppress Ras function. However, upon recruitment of Sos to the membrane, Sos binds Ras-GDP and facilitates release of GDP. In cells, the nucleotide GTP is about 10-fold more abundant than GDP; GTP binds to Ras by mass action. Ras-GTP adopts a conformation that permits interaction with downstream targets called effector molecules. These effectors include the protein kinase Raf, which activates the MAP kinase cascade; GTPase-activating protein, which links Ras to the Rho/Rac pathway; and phosphoinositide (PI) 3'-kinase and Ral-guanine nucleotide dissociation stimulator (Ral-GDS), which activate lipid pathways (2). The dysregulation of these signals in tumor cells leads to multiple cellular changes, including alterations in DNA synthesis, lipid metabolism, cellular morphology, cell adhesion properties, and gene expression.
In the broadest sense, the study of signaling mechanisms has already yielded therapeutic agents in the treatment of cancer, as evidenced by antiestrogens, antiandrogens, agonists of gonadotropin-releasing hormone, and stem cell growth factors, for example. However, research into oncoproteins that function within the signal transduction system is only beginning to be applied in the clinic. Therapeutic approaches of interest include tools such as mAbs against the extracellular domain of receptors, oligonucleotides that are antisense to key target proteins, and small molecule inhibitors of enzymes (Table 1).

\section{Growth factor receptors}

Efforts to inhibit HER-2 yielded the first cancer therapeutic agent based on research in growth factor signaling. Unlike other members of the EGF receptor family, HER-2 has no known ligand (3). HER-2 expression is upregulated in approximately $25-30 \%$ of human breast cancers; this upregulation is believed to promote HER-2 heterodimerization with other members of the EGF receptor family, as well as HER-2 homodimerization, which results in a constitutively active tyrosine kinase. Increased expression of HER-2 generally correlates with the severity of disease, and expression is consistently higher in tumor tissue than in normal tissue, making the tumor more prone to antibody therapy.

Genentech Inc. developed the mAb trastuzumab, which is directed against the extracellular domain of HER-2 (4). Use of this drug requires genotyping patient tumor samples for the expression of HER-2. It is thought that trastuzumab inhibits the proliferation of breast cancer cells by several mechanisms (5). First, binding of trastuzumab is associated with upregulation of the p27 $27^{\text {Kip }}$ inhibitor of some cyclin-dependent kinases. Second, this agent accelerates the internalization and degradation of HER-2, reducing the cellular level of activated tyrosine protein kinase. Third, trastuzumab may induce immune-mediated effects, including cell-mediated cytotoxicity and complement fixation. In combination with cisplatin, doxorubicin, and especially paclitaxel, trastuzumab shows enhanced anti-tumor activity in preclinical models (6). Trastuzumab has also proved its value in the clinic and is particularly effective in combination with paclitaxel $(7,8)$. The combination of trastuzumab with doxorubicin also appears to be effective, but may have higher cardiotoxicity than trastuzumab alone $(8,9)$.

From the perspective of pharmaceutical development, it is interesting to note that the time from the discovery of the HER-2/c-neu oncogene in 1985 and the association of HER-2 amplification in human breast cancer in 
Table 1

Examples of inhibitors of growth factor signaling for cancer treatment

\begin{tabular}{|c|c|c|c|}
\hline Target & Compound & Mechanism of action & Development status \\
\hline HER2/c-neu & Trastuzumab & $\mathrm{mAb}$ & Launched as Herceptin ${ }^{\mathrm{TM}}$ \\
\hline \multirow{5}{*}{ EGF receptor } & C225 & $\mathrm{mAb}$ & Phase III \\
\hline & E7.6.3 & $\mathrm{mAb}$ & Preclinical \\
\hline & ZD-1839 & Kinase inhibitor & Phase II \\
\hline & CP-358,774 & Kinase inhibitor & Phase II \\
\hline & PD-168,393 & Kinase inhibitor & Preclinical \\
\hline PDGF receptor & SU-101 & Kinase inhibitor & Phase III \\
\hline IGFR & AS ODN & Antisense & Preclinical \\
\hline \multirow{5}{*}{ Ras } & ISIS-2503 & Antisense & Phase II \\
\hline & R115777 & $\mathrm{FTI}$ & Phase II \\
\hline & SCH 66336 & $\mathrm{FTI}$ & Phase II \\
\hline & L-778,123 & $\mathrm{FTI}$ & Phase I \\
\hline & BMS-214662 & $\mathrm{FTI}$ & Phase I \\
\hline \multirow[t]{3}{*}{ Raf } & ISIS-5132 & Antisense & Phase II \\
\hline & ZM 336372 & Kinase inhibitor & Preclinical \\
\hline & L-779,450 & Kinase inhibitor & Preclinical \\
\hline \multirow[t]{2}{*}{ MEK } & PD-184352 & Kinase inhibitor & Preclinical \\
\hline & U0126 & Kinase inhibitor & Preclinical \\
\hline \multirow[t]{3}{*}{ PKC } & ISIS-3521 & Antisense & Phase II \\
\hline & CGP 41251 & Kinase inhibitor & Phase II \\
\hline & UCN-01 & Kinase inhibitor & Phase I \\
\hline PI 3'-kinase & LY 294002 & Kinase inhibitor & Preclinical \\
\hline
\end{tabular}

1987 to FDA approval of trastuzumab in 1998 was a relatively short period. This rapid progress reflects an understanding of the underlying science, as well as the fact that trastuzumab is a biological agent. In general, biological agents may be developed more quickly than are chemical entities.

Therapeutic antibodies have also been developed against the EGF receptor. C225, a human/mouse chimeric antibody (10), and E7.6.3, a fully human antibody (11), bind to the EGF receptor extracellular domain and block EGF ligand binding. These antibodies block the ligand-dependent proliferation of breast cancer cell lines in cell culture, and can induce tumor regression in mouse xenograft tumor assays. Like trastuzumab, C225 appears to be especially effective in combination with doxorubicin or paclitaxel (10). C225 is currently undergoing clinical evaluation. In preliminary trial results, complete responses were noted in head and neck cancers when C225 was combined with radiotherapy.

The EGF receptor is also the target for the development of inhibitors of the intracellular tyrosine kinase domain. ZD-1839 and CP-358,774, competitive inhibitors of ATP binding to the receptor's active site, are currently in clinical trials $(12,13)$. Their mechanism of action has led to some concern about safety, given the variety and physiological significance of protein kinases and other enzymes that bind ATP. However, these compounds appear to have good anti-cancer activity in preclinical models, with an acceptable therapeutic index, particularly in patients with non-small cell lung cancer. The dermatological toxicity observed for these drugs is most likely mechanism based, arising as a consequence of their intended biochemical activities. More recently, highly potent and selective irreversible inhibitors of the EGF receptor kinase have been reported, such as PD-168,393 (14). This compound appears to bind specifically to an active-site cysteine residue near the ATP binding site; its irreversible binding may afford improved anti-tumor activity. It will be interesting to monitor the development of this class of inhibitor: such reactive molecules are often dismissed as drugs, because of their potential for nonspecific interactions, but if they are sufficiently selective for their targets, reactivity need not be seen as a negative trait. Aspirin, for example, is an irreversible inhibitor of cyclooxygenases.

SU-101, an inhibitor of PDGF receptor kinase activity $(15,16)$, is currently in phase II development for treating glioblastomas. Another receptor tyrosine kinase that has been explored with increasing attention as a drug target is the IGF type I (IGF-I) receptor $(17,18)$. This receptor activates cell proliferation, but its role as an antiapoptotic signal may be more significant. Initial evidence from preclinical studies of an antisense oligonucleotide suggests that IGF-I receptor inhibition can promote tumor apoptosis (17).

\section{Targeting a GTPase switch}

The ras gene, discovered in 1978, has attracted a great deal of attention because it was the among the first oncogenes associated with human cancer, and studies of Ras function have helped to elucidate many of the mitogenic cell signaling pathways (19). Mutated forms of Kirsten-ras (Ki-ras) and N-ras are found in solid tumors (lung, colon, pancreas, and brain) and leukemias, whereas mutant Harvey-ras (Ha-ras) alleles are found in only a small subset of bladder, head, and neck tumors. The agents currently in clinical trials that are based on this area of research act either by regulating ras gene expression or by inhibiting protein farnesylation. An antisense oligonucleotide (ISIS-2503) directed against Ha-ras expression (20) displayed significant anti-tumor activity against a variety of human tumor cell lines in preclinical mouse tumor xenograft studies. ISIS-2503 appears to act against tumors whether or not they have suffered mutations in Ha-ras, but the basis of this broad activity is unclear. ISIS-2503 has completed phase I evaluation; an initial report noted some disease stabilization when this agent was administered by continuous intravenous infusion (20).

A second approach for inhibiting Ras function has attracted broad attention within the pharmaceutical industry. Ras proteins carry an essential lipid moiety - a farnesyl group - at their $\mathrm{COOH}$ termini. Genetic data indicate that inhibition of Ras farnesylation blocks Ras localization to the plasma membrane. Without this membrane localization, Ras fails to interact with critical regulatory and effector molecules (19), and is transformation defective. Hence, farnesyl-protein transferase inhibitors (FTIs) are predicted to block cellular transformation. 
However, the transferase reaction is essential not only to the function of Ras, but also to the function of at least 20 other farnesyl proteins. Thus, FTIs are not truly Ras-specific inhibitors. Nevertheless, a number of FTIs have been developed as potential anti-cancer drugs $(21,22)$.

Potent FTIs of diverse chemical structures inhibit tumor growth in both nude mouse xenograft models and a variety of transgenic mouse tumor models - including those that overexpress Ha-ras, Ki-ras, or N-ras (21). The similar effects of structurally distinct FTIs, and their effectiveness at doses that block substrate protein farnesylation, confirm that these compounds achieve the desired anti-tumor activity by inhibiting farnesyl-protein transferase. Unlike cytotoxic anti-tumor agents, FTIs appear to act without overt systemic toxicity. Since FTIs were originally thought to be cytostatic agents, it was surprising to observe in preclinical tissue culture and transgenic tumor models that they induce apoptosis in tumor cells. The induction of apoptosis occurs by caspase- 3 activation and is independent of wild-type p53 function (21, 23) - an important finding given the usual association of loss of p53 function with resistance to chemotherapy (see Sellers and Fisher in this Perspective series).

In 1997 and 1998, nearly 20 years after the discovery of Ras and about 9 years after the discovery of Ras farnesylation, clinical trials began with FTIs (22). At least 4 different FTIs are currently undergoing evaluation: R115777; SCH 66336; L-778,123; and BMS-214662 (24) (Table1). R115777 and SCH 66336 are administered by the oral route, L-778,123 is given by continuous infusion, and BMS-214662 is administered either orally or intravenously. The more advanced trials with R115777 and SCH 66336 have reported dose-limiting toxicities involving bone marrow and the gastrointestinal tract, indicating that at high enough concentrations, FTIs can have general antiproliferative effects on normal tissues. The doses achieved in the clinic so far with L-778,123 and SCH 66336 were sufficient to inhibit protein farnesylation in readily obtainable tissues such as white blood cells and cells of the buccal mucosa. Reports on the efficacy of FTIs are anxiously awaited. Based upon preclinical data, it is anticipated that FTIs will also be used in combination with other treatments, such as paclitaxel, vincristine, cisplatin, 5-fluorouracil, gemcitabine, cyclophosphamide, or radiation (25-28).

\section{Inhibiting protein kinase effectors}

A series of protein phosphorylation events within the cell ensue upon Ras activation. The first key step is the direct binding of the Raf protein kinase to Ras-GTP $(1,2)$. Raf in turn phosphorylates and activates MAP/Erk kinase (MEK), which in turn phosphorylates and activates MAP kinase. The key role of this pathway in Ras-mediated cellular transformation has inspired several efforts to develop inhibitors of these protein kinase reactions (Table 1). ISIS-5132, an antisense oligonucleotide directed against Raf, is in phase II clinical development (20). This compound causes a dose-dependent reduction of c-Raf mRNA levels in preclinical tumor models. This pharmacodynamic monitoring has also been performed in the clinic using peripheral blood mononuclear cells from treated patients as a tissue source. In a phase I trial, the median reduction of Raf mRNA was $42 \%$ at 48 hours, with significant inhibitions observed up to 15 days, although this decrease did not appear to be dose dependent. Of the 65 patients evaluated in these initial reports, 4 patients with ovarian, pancreatic, renal, and colon cancer have seen their disease remain stable for up to 10 months. Interestingly, in 2 of the other patients, disease progression coincided with the loss of suppression of Raf mRNA levels (20).

Raf protein kinase inhibitors remain at an earlier stage of development. The most extensive analysis is from HallJackson et al. $(29,30)$, who characterized the biological effects of both a direct Raf kinase inhibitor, ZM 336372, and a p38 kinase inhibitor, SB 203580, which weakly inhibits Raf kinase activity. Cells treated with ZM 336372 or SB 203580 exhibit a paradoxical increase in Raf activity measured ex vivo, indicating that these compounds do not inhibit Raf signaling pathways. ZM 336372 does not inhibit Ras- or Raf-mediated cellular transformation, but a preliminary report by Heimbrook et al. (31) indicates that the triarylimidazole derivative L-779,450, which inhibits Raf protein kinase activity in vitro, blocks intracellular signaling by Ki-Ras and Ha-Ras.

Two groups have recently described novel MEK inhibitors (Table 1). Parke-Davis Pharmaceutical Research, which described the first MEK inhibitor, PD098059, identified a more potent and selective compound (PD-184352) from a coupled biochemical screen that included GST-MEK, MAP kinase, and the MAP kinase substrate myelin basic protein (32). DuPont Pharmaceuticals Co. identified U0126 in a cell-based assay that monitored AP-1 response elements, and they subsequently found that this compound inhibits MEK activity (33). Neither PD-184352 nor U0126 compete for binding to ATP or protein substrates, suggesting that these compounds function as allosteric inhibitors of MEK. Both compounds block MAP kinase phosphorylation in cells, and at doses that abolish intracellular MEK activity, PD-184352 inhibits the anchorage-independent growth of several human tumor cell lines and causes cells to adopt a flattened morphology. At similar doses, PD-184352 also inhibited tumor growth in mouse tumor xenograft models (32). The correlation between this surrogate biochemical endpoint and biological activity provides strong evidence for mechanismbased anti-tumor activity, but MEK inhibitors remain at the preclinical development stage.

\section{Blocking lipid-mediated signaling}

Activation of growth factor receptors is also associated with changes in phospholipid metabolism $(1-3,18)$. In 1 pathway, the phosphorylated residues on the intracellular domain of these receptors bind phospholipase C, which then cleaves membrane phospholipids. One of these breakdown products, diacylglycerol, can activate some forms of protein kinase C (PKC), such as PKC- $\alpha$, which has been implicated in cell proliferative processes and tumorigenesis (34). PKC- $\alpha$ expression has been found in some human breast tumors to be elevated relative to surrounding normal tissue. Both antisense inhibitors to PKC- $\alpha$ (ISIS-3521) and inhibitors of PKC kinase activity (CGP 41251 and $\mathrm{UCN}-01$ ) are in clinical 
trials (Table 1). The kinase inhibitors, both of which are derivatives of staurosporine, potently inhibit PKC activity and are active in mouse tumor xenograft models (34). CGP 41251 also inhibits the P-glycoprotein transporter, which mediates the multidrug resistance of many advanced tumors. The toxicities noted for UCN-01 and CGP 41251 in the clinic are so far not remarkable, but this may be related to the high capacity of these compounds to bind plasma proteins - a characteristic that might also be expected to blunt their anti-tumor activity (34). The antisense compound ISIS-3521 exhibits an acceptable safety profile. Its side effects - fatigue, fever, and thrombocytopenia - are typical of phosphorothioate-based antisense compounds (20). ISIS-3521 is being tested in combination with carboplatin and paclitaxel in patients with non-small cell lung cancer; preliminary data indicate partial responses in 6 of 8 patients treated.

In a second pathway, activation of Ras directly activates PI 3 '-kinase. The product of this reaction is then able to activate the protein kinase Akt, which is a suppressor of apoptosis (2). Inhibition of PI 3'-kinase activity would then be predicted to inactivate Akt activity and subsequently activate apoptotic pathways in tumors. In preclinical studies, LY 294002 potently inhibited PI 3'kinase. This compound inhibits lipid signaling by growth factor receptors. In combination with an FTI, it was shown to induce apoptosis in attached tumor cells, which normally do not respond to FTI alone (35). This result raises the interesting possibility that inhibitors of different steps of the signaling pathways may be of greatest benefit when used in combination.

\section{Conclusions}

Growth factor-regulated proliferation pathways elucidated over the last 2 decades are finally reaching the clinic to be tested. So far, just 1 product, trastuzumab, has emerged, but its apparent success provides much encouragement. This product shows the therapeutic value of a treatment based upon a fundamental genetic defect in a cancer and raises hopes for other agents, such as those summarized in Table 1. It is interesting to note how our thinking has changed as the basic research findings of growth factor signaling have been translated into pharmaceutical entities.

First, it has become clear that these compounds do not act solely on tumor tissue. Each agent has a particular toxicity that must be managed. In some cases, as with EGF receptor inhibitors or FTIs, these effects are mechanism based, but the undesirable consequences of other agents, including phosphorothioate antisense oligonucleotide compounds, are structure based. In either event, therapies developed on growth signaling pathways offer new mechanisms to attack cancer, but they do not necessarily provide a true cure for cancer.

Second, we have come to appreciate the value of combining these new inhibitors with existing therapeutic regimens. This realization reinforces the notion that cancer is a disease of multiple and changing genetic alterations that must be attacked with therapies having different mechanisms of action. Therapies designed based on knowledge of signal transduction pathways represent just 1 approach to developing new agents. Clearly, simi- lar rational molecular approaches for anti-cancer therapies may also be developed to control cell cycle regulation and cell cycle checkpoints (see Shapiro in this Perspective series), apoptosis (Sellers and Fisher, this series), telomere biology, and angiogenesis (Keshet, this series). How these different therapeutic strategies can best be combined remains an open question. Will it be better to have multiple inhibitors targeting different steps of growth factor signaling pathways? Or will agents directed at different fundamental aspects of a cancer cell prove the most effective combination?

Finally, it is interesting to note that surrogate pharmacodynamic endpoints are beginning to be used for the development of signal transduction inhibitors (see Druker and Lydon in this Perspective series). In preclinical animal models, the biological efficacy of FTIs was monitored in relation to inhibition of protein farnesylation and inhibition of downstream pathways such as MAP kinase and p70 kinase. Likewise, inhibition of MAP kinase phosphorylation showed a positive correlation with the anti-tumor activity of the MEK inhibitor PD-184352. Development of the ISIS antisense compounds has also been linked with a reduction in the target mRNA levels. This approach has also been carried into the clinical development of some of these compounds, such as has been reported for SCH 66336, L778,123 , and ISIS-5132. Given the genetic complexities of cancer, it will be important to analyze whether monitoring these pharmacodynamic endpoints provides useful clinical information, particularly for compounds that do not have clearly defined dose-limiting toxicities. After all, this is what some believe to be the ultimate promise of these agents: lethality to tumors without overt systemic toxicity.

1. Heimbrook, D., Oliff, A., and Gibbs, J.B. 1997. Essentials of signal transduction. 5th edition. Lippincott-Raven Publishers. Philadelphia, PA. 35-45.

2. Vojtek, A.B., and Der Channing, J. 1998. Increasing complexity of the Ras signaling pathway. J. Biol. Chem. 273:19925-19928.

3. Hung, M.C., and Lau, Y.K. 1999. Basic science of HER-2/neu: a review. Semin. Oncol. 26:51-59.

4. Goldenberg, M.M. 1999. Trastuzumab, a recombinant DNA-derived humanized monoclonal antibody, a novel agent for the treatment of metastatic breast cancer. Clin. Ther. 21:309-318.

5. Sliwkowski, M.X., et al. 1999. Nonclinical studies addressing the mechanism of action of trastuzumab (Herceptin). Semin. Oncol. 26:60-70.

6. Baselga, J., Norton, L., Albanell, J., Kim, Y.M., and Mendelsohn, J. 1998. Recombinant humanized anti-HER2 antibody (Herceptin) enhances the antitumor activity of paclitaxel and doxorubicin against HER2/neu overexpressing human breast cancer xenografts. Cancer Res. 58:2825-2831.

7. Baselga, J., et al. 1999. Phase II study of weekly intravenous trastuzum$\mathrm{ab}$ (Herceptin) in patients with HER2/neu-overexpressing metastatic breast cancer. Semin. Oncol. 26:78-83.

8. Shak, S. 1999. Overview of the trastuzumab (Herceptin) anti-HER2 monoclonal antibody clinical program in HER2-overexpressing metastatic breast cancer. Herceptin Multinational Investigator Study Group Semin. Oncol. 26:71-77.

9. Ewer, M.S., Gibbs, H.R., Swafford, J., and Benjamin, R.S. 1999. Cardiotoxicity in patients receiving transtuzumab (Herceptin): primary toxicity, synergistic or sequential stress, or surveillance artifact? Semin. Oncol. 26:96-101.

10. Fan, Z., and Mendelsohn, J. 1998. Therapeutic application of antigrowth factor receptor antibodies. Curr. Opin. Oncol. 10:67-73.

11. Yang, X.D., et al. 1999. Eradication of established tumors by a fully human monoclonal antibody to the epidermal growth factor receptor without concomitant chemotherapy. Cancer Res. 59:1236-1243.

12. Woodburn, J.R., et al. 1997. ZD1839, an epidermal growth factor tyrosine kinase inhibitor selected for clinical development. Proceedings of the American Association for Cancer Research Annual Meeting. 38:633.

13. Moyer, J.D., et al. 1997. Induction of apoptosis and cell cycle arrest by CP-358,774, an inhibitor of epidermal growth factor receptor tyrosine 
kinase. Cancer Res. 57:4838-4848.

14. Singh, J., et al. 1997. Structure-based design of a potent, selective, and irreversible inhibitor of the catalytic domain of the erbB receptor subfamily of protein tyrosine kinases. J. Med. Chem. 40:1130-1135.

15. Eckhardt, S.G., et al. 1999. Phase I and pharmacologic study of the tyrosine kinase inhibitor SU101 in patients with advanced solid tumors. $J$. Clin. Oncol. 17:1095-1104.

16. Shawver, L.K., et al. 1996. SU101, a potent inhibitor of PDGF-mediated signaling, inhibits growth of a wide variety of tumor types in vivo. Proceedings of the American Association for Cancer Research Annual Meeting. 37:399.

17. Baserga, R., Hongo, A., Rubini, M., Prisco, M., and Valentinis, B. 1997. The IGF-I receptor in cell growth, transformation, and apoptosis. Biochim. Biophys. Acta. 1332:F105-F126.

18. Petley, T., Graff, K., Jiang, W., Yang, H., and Florini, J. 1999. Variation among cell types in the signaling pathways by which IGF-I stimulates specific cellular responses. Horm. Metab. Res. 31:70-76.

19. Lowy, D.R., and Willumsen, B.M. 1993. Function and regulation of Ras. Annu. Rev. Biochem. 62:851-891.

20. Holmlund, J.T., Monia, B.P., Kwoh, T.J., and Dorr, F.A. 1999. Toward antisense oligonucleotide therapy for cancer: ISIS compounds in clinical development. Current Opinion in Molecular Therapeutics. 1:372-385.

21. Oliff, A. 1999. Farnesyltransferase inhibitors: targeting the molecular basis of cancer. Biochim. Biophys. Acta. 1423:C19-C30.

22. Williams, T.M. 1999. Inhibitors of protein prenylation. Expert opinion on therapeutic patents. 9:1263-1280.

23. Suzuki, N., Urano, J., and Tamanoi, F. 1998. Farnesyltransferase inhibitors induce cytochrome $\mathrm{c}$ release and caspase 3 activation preferentially in transformed cells. Proc. Natl. Acad. Sci. USA. 95:15356-15361.

24. Ferrante, K., Winograd, B., and Canetta, R. 1999. Promising new developments in cancer chemotherapy. Cancer Chemother. Pharmacol.
43(Suppl.):61-68

25. Moasser, M.M., et al. 1998. Farnesyl transferase inhibitors cause enhanced mitotic sensitivity to taxol and epothilones. Proc. Natl. Acad. Sci. USA. 95: 1369-1374.

26. Muschel, R.J., Soto, D.E., McKenna, W.G., and Bernhard, E.J. 1998. Radiosensitization and apoptosis. Oncogene. 17:3359-3363.

27. Liu, M., et al. 1998. Antitumor activity of SCH 66336, an orally bioavailable tricyclic inhibitor of farnesyl protein transferase, in human tumor xenograft models and wap-ras transgenic mice. Cancer Res. 58:4947-4956.

28. Sun, J.Z., et al. 1999. Antitumor efficacy of a novel class of non-thiol-containing peptidomimetic inhibitors of farnesyltransferase and geranylgeranyltransferase I: combination therapy with the cytotoxic agents cisplatin, Taxol, and gemcitabine. Cancer Res. 59:4919-4926.

29. Hall-Jackson, C.A., et al. 1999. Paradoxical activation of Raf by a novel Raf inhibitor. Chem. Biol. 6:559-568.

30. Hall-Jackson, C.A., Goedert, M., Hedge, P., and Cohen, P. 1999. Effect of SB 203580 on the activity of c-Raf in vitro and in vivo. Oncogene. 18:2047-2054.

31. Heimbrook, D.C., et al. 1998. Identification of potent, selective kinase inhibitors of Raf. Proceedings of the American Association for Cancer Research Annual Meeting. March. 39:558.

32. Sebolt-Leopold, J.S., et al. 1999. Blockade of the MAP kinase pathway suppresses growth of colon tumors in vivo. Nat. Med. 5:810-816.

33. Favata, M.F., et al. 1998. Identification of a novel inhibitor of mitogenactivated protein kinase kinase. J. Biol. Chem. 273:18623-18632.

34. Gescher, A. 1998. Analogs of staurosporine: potential anticancer drugs? Gen. Pharmacol. 31:721-728.

35. Du, W., Liu, A., and Prendergast, G.C. 1999. Activation of the PI3'K-AKT pathway masks the proapoptotic effects of farnesyltransferase inhibitors. Cancer Res. 59:4208-4212. 